\title{
Signless Laplacian Spectral Conditions for Hamiltonicity of Graphs
}

\author{
Guidong Yu, ${ }^{1}$ Miaolin Ye, ${ }^{1}$ Gaixiang Cai, ${ }^{1}$ and Jinde $\mathrm{Cao}^{2,3}$ \\ ${ }^{1}$ School of Mathematics \& Computation Sciences, Anqing Normal College, Anqing 246011, China \\ ${ }^{2}$ Department of Mathematics, Southeast University, Nanjing, Jiangsu 210096, China \\ ${ }^{3}$ Department of Mathematics, Faculty of Science, King Abdulaziz University, Jeddah 21589, Saudi Arabia
}

Correspondence should be addressed to Jinde Cao; jdcao@seu.edu.cn

Received 23 January 2014; Revised 7 June 2014; Accepted 16 June 2014; Published 30 June 2014

Academic Editor: Francesco Soldovieri

Copyright (C) 2014 Guidong Yu et al. This is an open access article distributed under the Creative Commons Attribution License, which permits unrestricted use, distribution, and reproduction in any medium, provided the original work is properly cited.

We establish some signless Laplacian spectral radius conditions for a graph to be Hamiltonian or traceable or Hamilton-connected.

\section{Introduction}

Let a graph, $G=(V, E)$ be a simple graph of order $n$ with vertex set $V=\left\{v_{1}, v_{2}, \ldots, v_{n}\right\}$ and edge set $E$. Denote by $e(G):=|E|$ the number of edges of the graph $G$. Write by $K_{n}$ a complete graph of order $n, O_{n}$ an empty graph of order $n$ (without edges), and $K_{n, m}$ a complete bipartite graph with two parts having $n, m$ vertices, respectively. The graph $G$ is said to be Hamiltonian, if it has a Hamiltonian cycle which is a cycle of order $n$ contained in $G$. The graph $G$ is said to be traceable if it has a Hamiltonian path which is a path of order $n$ contained in $G$. The problem of deciding whether a graph is Hamiltonian is Hamiltonian problem, which is one of the most difficult classical problems in graph theory. Indeed, it is NP-complete problem.

The adjacency matrix of $G$ is defined to be a matrix $A(G)=\left[a_{i j}\right]_{n \times n}$, where $a_{i j}=1$ if $v_{i}$ is adjacent to $v_{j}$ and $a_{i j}=0$ otherwise. The largest eigenvalue of $A(G)$ is called to be the spectral radius of $G$, which is denoted by $\mu(G)$. The degree matrix of $G$ is written by $D(G)=$ $\operatorname{diag}\left(d_{G}\left(v_{1}\right), d_{G}\left(v_{2}\right), \ldots, d_{G}\left(v_{n}\right)\right)$, where $d_{G}\left(v_{i}\right)(i=1,2, \ldots$, $n)$ denotes the degree of the vertex $v_{i}$ in the graph $G$. The signless Laplacian matrix of $G$ is defined by $Q(G)=D(G)+$ $A(G)$. The largest eigenvalue of $Q(G)$ is called to be the signless Laplacian spectral radius of $G$, which is denoted by $q(G)$.

Recently, using spectral graph theory to study the Hamiltonian problem has received a lot of attention. Some spectral conditions for a graph to be Hamiltonian or traceable have been given in [1-6]. In this paper, we still study the Hamiltonicity of a graph. Firstly, we present a signless Laplacian spectral radius condition for a bipartite graph to be Hamiltonian in Section 2. Secondly, we give some signless Laplacian spectral radius conditions for a graph to be traceable or Hamilton-connected in Section 3 and Section 4, respectively.

\section{Signless Laplacian Spectral Radius in Hamiltonian Bipartite Graphs}

The definition of the closure of a balanced bipartite graph can be found in $[7,8]$. For a positive integer $k$, the $k$-closure of a balanced bipartite graph $G_{B P T}:=(X, Y ; E)$, where $|X|=$ $|Y|$, written by $\mathscr{C}_{k}\left(G_{B P T}\right)$, is a graph obtained from $G_{B P T}$ by successively joining pairs of nonadjacent vertices $x \in X$ and $y \in Y$, whose degree sum is at least $k$, until no such pairs remain. By the definition of the $\mathscr{C}_{k}\left(G_{B P T}\right)$, we have that $d_{\mathscr{C}_{k}\left(G_{B D T}\right)}(x)+d_{\mathscr{C}_{k}\left(G_{B P T}\right)}(y) \leq k-1$ for any pair of nonadjacent vertices $x \in X$ and $y \in Y$ of $\mathscr{C}_{k}\left(G_{B P T}\right)$.

Lemma 1 (see [9]). Let $G_{B P T}=(X, Y ; E)$ be a connected balanced bipartite graph, where $|X|=|Y|=r \geq 2$. Then, $G_{B P T}$ is Hamiltonian if and only if $\mathscr{C}_{r+1}\left(G_{B P T}\right)$ is Hamiltonian.

For a graph $G$, write $Z(G):=\sum_{u v \in E(G)}\left(d_{G}(u)+d_{G}(v)\right)=$ $\sum_{u \in V(G)} d_{G}^{2}(u)$, and let $\Delta(G)$ be maximum degree of $G$. A 
regular graph is a graph for which every vertex in the graph has the same degree. A semi-regular graph is a bipartite graph for which every vertex in the same partite set has the same degree.

Lemma 2 (see [2]). Let $G$ be a graph with at least one edge. Then,

$$
q(G) \geq \frac{Z(G)}{e(G)}
$$

if and only if $G$ is regular or semi-regular.

Let $M$ be a Hermitian matrix of order $n$, and let $\lambda_{1}(M) \geq$ $\lambda_{2}(M) \geq \cdots \geq \lambda_{n}(M)$ be the eigenvalues of $M$.

Lemma 3 (see [10]). Let $B$ and $C$ be Hermitian matrices of order $n, 1 \leq i, j \leq n$. Then,

$$
\lambda_{i}(B)+\lambda_{j}(C) \geq \lambda_{i+j-1}(B+C),
$$

if $i+j \leq n+1$.

Lemma 4. Let $G$ be a graph. Then,

$$
q(G) \leq \mu(G)+\Delta(G) .
$$

Proof. Because $Q(G)=A(G)+D(G)$, by Lemma 3,

$$
\lambda_{1}(A(G))+\lambda_{1}(D(G)) \geq \lambda_{1}(Q(G)) .
$$

We notice that $\lambda_{1}(A(G))=\mu(G), \lambda_{1}(D(G))=\Delta(G)$, and $\lambda_{1}(Q(G))=q(G)$. So, the result follows.

Let $G_{B P T}=(X, Y ; E)$ be a bipartite graph, the quasicomplement of $G_{B P T}$ is denoted by $G_{B P T}^{*}:=\left(X, Y ; E^{\prime}\right)$, where $E^{\prime}=\{x y: x \in X, y \in Y, x y \notin E\}$.

Theorem 5. Let $G_{B P T}=(X, Y ; E)$ be a connected balanced bipartite graph, where $|X|=|Y|=r \geq 2$. If

$$
q\left(G_{B P T}^{*}\right)<r
$$

then $G_{B P T}$ is Hamiltonian.

Proof. Suppose that $G_{B P T}$ is not Hamiltonian. Then, $H_{B P T}:=$ $\mathscr{C}_{r+1}\left(G_{B P T}\right)$ is not Hamiltonian too by Lemma 1, and therefore, $H_{B P T}$ is not $K_{r, r}$. Thus, there exists a vertex $x \in X$ and a vertex $y \in Y$ such that $x y \notin E\left(H_{B P T}\right)$. We find that $d_{H_{B P T}}(x)+d_{H_{B P T}}(y) \leq r$ for any pair of nonadjacent vertices $x \in X$ and $y \in Y$ in $H_{B P T}$. So,

$$
d_{H_{B P T}^{*}}(x)+d_{H_{B P T}^{*}}(y)=r-d_{H_{B P T}}(x)+r-d_{H_{B P T}}(y) \geq r,
$$

for any pair of adjacent vertices $x \in X, y \in Y$ in $H_{B P T}^{*}$. Hence,

$$
Z\left(H_{B P T}^{*}\right)=\sum_{x y \in E\left(H_{B P T}^{*}\right)}\left(d_{H_{B P T}^{*}}(x)+d_{H_{B P T}^{*}}(y)\right) \geq \operatorname{re}\left(H_{B P T}^{*}\right) .
$$

By Lemma 2, we have that

$$
q\left(H_{B P T}^{*}\right) \geq \frac{Z\left(H_{B P T}^{*}\right)}{e\left(H_{B P T}^{*}\right)} \geq r .
$$

As $H_{B P T}^{*}$ is a subgraph of $G_{B P T}^{*}$, by Perron-Frobenius theorem,

$$
q\left(G_{B P T}^{*}\right) \geq q\left(H_{B P T}^{*}\right) .
$$

Thus, by (5), (8), and (9), we have that

$$
r>q\left(G_{B P T}^{*}\right) \geq q\left(H_{B P T}^{*}\right) \geq \frac{Z\left(H_{B P T}^{*}\right)}{e\left(H_{B P T}^{*}\right)} \geq r,
$$

a contradiction.

Li [4] has given a sufficient condition for a bipartite graph to be Hamiltonian as follows.

Theorem 6 (see [4]). Let $G_{B P T}=(X, Y ; E)$ be a connected balanced bipartite graph, where $|X|=|Y|=r \geq 2$. If

$$
\mu\left(G_{B P T}^{*}\right) \leq \sqrt{\frac{r-2}{2}},
$$

then $G_{B P T}$ is Hamiltonian.

Remark 7. We now compare Theorems 5 and 6. If $\mu\left(G_{B P T}^{*}\right) \leq$ $\sqrt{(r-2) / 2}$ and $\Delta\left(G_{B P T}^{*}\right)<r-\sqrt{(r-2) / 2}$, we have that $q\left(G_{B P T}^{*}\right)<r$ by Lemma 4. Hence Theorem 5 improves Theorem 6 when $\Delta\left(G_{B P T}^{*}\right)<r-\sqrt{(r-2) / 2}$. For example, let $G_{B P T}$ be a regular connected balanced bipartite graph with degree $(r+1) / 2$, where $r$ is odd and $|X|=|Y|=r \geq 6$. Then, its quasi-complement $G_{B P T}^{*}$ is a regular graph with degrees $(r-$ $1) / 2, \mu\left(G_{B P T}^{*}\right)=(r-1) / 2$, and $q\left(G_{B P T}^{*}\right)=r-1 . G_{B P T}$ satisfies the condition of Theorems 5 , and hence, it is Hamiltonian. But it does not satisfy the condition of Theorem 6 .

\section{Signless Laplacian Spectral Radius in Traceable Graphs}

Write $K_{n-1}+v$ for $K_{n-1}$ together with an isolated vertex. Let $G=(V(G), E(G))$ and $H=(V(H), E(H))$ be two disjoint graphs. The disjoint union of $G$ and $H$, denoted by $G \cup H$, is the graph with vertex set $V(G) \cup V(H)$ and edge set $E(G) \cup$ $E(H)$. If $G_{1} \cong \cdots \cong G_{k}$, we write $k G_{1}$ for $G_{1} \cup \cdots \cup G_{k}$. The join of $G$ and $H$, denoted by $G \vee H$, is the graph obtained from $G \cup H$ by adding edges joining every vertex of $G$ to every vertex of $H$.

Lemma 8 (see [3]). Let $G$ be a connected graph of order $n \geq 4$. If

$$
e(G) \geq \frac{(n-2)(n-3)}{2}+2,
$$

then $G$ is traceable unless $G \cong K_{1} \vee\left(K_{n-3} \cup 2 K_{1}\right), K_{2} \vee\left(3 K_{1} \cup\right.$ $\left.K_{2}\right)$, or $K_{4} \vee\left(6 K_{1}\right)$. 
Let $G$ be a graph containing a vertex $v$. Denote $m_{G}(v)=$ $m(v)=\left(1 / d_{G}(v)\right) \sum_{u \in N_{G}(v)} d_{G}(u)$ if $d_{G}(v)>0$ and $m_{G}(v)=0$ otherwise, where $N_{G}(v)$ or simply $N(v)$ denotes the neighborhood of $v$ in $G$.

Lemma 9 (see [11]). Let $G$ be a graph of order $n$. Then,

$$
\max \left\{d_{G}(v)+m_{G}(v): v \in V(G)\right\} \leq \frac{2 e(G)}{n-1}+n-2,
$$

with equality if and only if $G \supseteq K_{1, n-1}$ or $G=K_{n-1}+v$.

Lemma 10 (see [12]). Let $G$ be a connected graph. Then

$$
q(G) \leq \max \left\{d_{G}(v)+m_{G}(v): v \in V(G)\right\},
$$

with equality if and only if $G$ is a regular graph or a semi-regular graph.

In fact, if $G$ is disconnected, there exists a component $H$ of $G$ such that

$$
\begin{aligned}
q(G) & =q(H) \leq \max \left\{d_{H}(v)+m_{H}(v): v \in V(H)\right\} \\
& \leq \max \left\{d_{G}(v)+m_{G}(v): v \in V(G)\right\} .
\end{aligned}
$$

So the inequality (14) also holds when $G$ is a disconnected graph. By Lemmas 9 and 10, we have the following result; also see [13].

Corollary 11. Let $G$ be a graph of order $n$. Then,

$$
q(G) \leq \frac{2 e(G)}{n-1}+n-2 .
$$

If $G$ is connected, then the equality in (16) holds if and only if $G=K_{1, n-1}$ or $G=K_{n}$. Otherwise, the equality in (16) holds if and only if $G=K_{n-1}+v$.

Given a graph $G$ of order $n$, a vector $X \in \mathbb{R}^{n}$ is called a function defined on $G$, if there is a 1-1 map $\varphi$ from $V(G)$ to the entries of $X$, simply written as $X_{u}=\varphi(u)$ for each $u \in V(G), X_{u}$ is also called the value of $u$ given by $X$. If $X$ is an eigenvector of $Q(G)$ corresponding to the eigenvalue $q$, then $X$ is defined naturally on $G$; that is, $X_{u}$ is the entry of $X$ corresponding to the vertex $u$. One can find that

$$
\left[q-d_{G}(v)\right] X_{v}=\sum_{u \in N_{G}(v)} X_{u}, \quad \text { for each } v \in V(G),
$$

where $N_{G}(v)$ denotes the neighborhood of $v$ in $G$. The equation (17) is called $(q, X)$-eigenequation of $G$.

Theorem 12. Let $G$ be a connected graph of order $n \geq 4$. If

$$
q(G) \geq \frac{2(n-2)^{2}+4}{n-1}
$$

then $G$ is traceable.

Proof. By Corollary 11 and (18), we have

$$
e(G) \geq \frac{(n-1) q(G)-(n-1)(n-2)}{2} \geq \frac{(n-2)(n-3)}{2}+2 .
$$

Suppose that $G$ is non-traceable. Then, by Lemma 8 and (19), $G \cong K_{1} \vee\left(K_{n-3} \cup 2 K_{1}\right), K_{2} \vee\left(3 K_{1} \cup K_{2}\right)$, or $K_{4} \vee\left(6 K_{1}\right)$.

If $G \cong K_{1} \vee\left(K_{n-3} \cup 2 K_{1}\right)$, let $X=\left(X_{1}, X_{2}, \ldots, X_{n}\right)^{T}$ be the eigenvector of $Q(G)$ corresponding to eigenvalue $q(G)$. By (18), we know that $q(G) \neq 1, n-4$. Thus, by (17), all vertices of degree 1 have the same values given by $X$, say $X_{1}$; all vertices of degree $n-3$ have the same values by $X$, say $X_{2}$. Denote by $X_{3}$ the value of the vertex of degree $n-1$ given by $X$. Also, by (17), we have

$$
\begin{gathered}
(q(G)-1) X_{1}=X_{3}, \\
(q(G)-(n-3)) X_{2}=(n-4) X_{2}+X_{3}, \\
(q(G)-(n-1)) X_{3}=2 X_{1}+(n-3) X_{2} .
\end{gathered}
$$

Transform (20) into a matrix equation $(B-q(G) \mathbf{I}) X^{\prime}=0$, where $X^{\prime}=\left(X_{1}, X_{2}, X_{3}\right)^{T}$ and

$$
B=\left[\begin{array}{ccc}
1 & 0 & 1 \\
0 & 2 n-7 & 1 \\
2 & n-3 & n-1
\end{array}\right] .
$$

Thus, $q(G)$ is the largest root of the following equation:

$$
q^{3}+(-3 n+7) q^{2}+\left(2 n^{2}-7 n\right) q-2 n^{2}+14 n-24=0 .
$$

Let $f(x)=x^{3}+(-3 n+7) x^{2}+\left(2 n^{2}-7 n\right) x-2 n^{2}+14 n-24$; then $f^{\prime}(x)=3 x^{2}+2(-3 n+7) x+2 n^{2}-7 n$. Let $f^{\prime}(x)=0$; we have two values $x_{1}$ and $x_{2}$, such that $f^{\prime}\left(x_{1}\right)=f^{\prime}\left(x_{2}\right)=0$, where

$$
\begin{aligned}
& x_{1}=\frac{3 n-7-\sqrt{3 n^{2}-21 n+49}}{3}, \\
& x_{2}=\frac{3 n-7+\sqrt{3 n^{2}-21 n+49}}{3} .
\end{aligned}
$$

Hence, $f(x)$ is strictly increasing with respect to $x$ for $x>$ $x_{2}$.

Because $f(2(n-3))=2 n^{2}-17 n+33>0$ and $\left(2(n-2)^{2}+\right.$ $4) /(n-1)>2(n-3)>x_{2}$, we have that $f\left(\left(2(n-2)^{2}+4\right) /(n-\right.$ 1)) $>0$, which implies that $q(G)<\left(2(n-2)^{2}+4\right) /(n-1)$.

If $G \cong K_{2} \vee\left(3 K_{1} \cup K_{2}\right)$, let $X=\left(X_{1}, X_{2}, \ldots, X_{7}\right)^{T}$ be the eigenvector of $Q(G)$ corresponding to eigenvalue $q(G)$. By (18), we know that $q(G) \neq 2,5$. Thus, by (17), three vertices of degree 2 have the same values given by $X$, say $X_{1}$; two vertices of degree 3 have the same values, say $X_{2}$; two vertices of degree 6 have the same values, say $X_{3}$. Also, by (17), we have

$$
\begin{gathered}
(q(G)-2) X_{1}=2 X_{3}, \\
(q(G)-3) X_{2}=X_{2}+2 X_{3}, \\
(q(G)-6) X_{3}=3 X_{1}+2 X_{2}+X_{3} .
\end{gathered}
$$

Transform (24) into a matrix equation $(B-q(G) \mathbf{I}) X^{\prime}=0$, where $X^{\prime}=\left(X_{1}, X_{2}, X_{3}\right)^{T}$ and

$$
B=\left[\begin{array}{lll}
2 & 0 & 2 \\
0 & 4 & 2 \\
3 & 2 & 7
\end{array}\right] .
$$


Thus, $q(G)$ is the largest root of the following equation:

$$
q^{3}-13 q^{2}+40 q-24=0
$$

Let $g(x)=x^{3}-13 x^{2}+40 x-24$; we can easily get that $g(x)$ is strictly increasing with respect to $x$ for $x>20 / 3$.

Consider $g(9)=12>0$, which implies that $q(G)<9$. $44 / 3$.

If $G \cong K_{4} \vee\left(6 K_{1}\right)$, we easily calculate $q(G)=8+2 \sqrt{10}<$

Thus, in either case, we have a contradiction.

Lu et al. [3] have given a sufficient condition for a graph to be traceable as follows.

Theorem 13 (see [3]). Let $G$ be a connected graph of order $n \geq$ 5. If

$$
\mu(G) \geq \sqrt{(n-3)^{2}+2},
$$

then $G$ is traceable.

Example 14. There are graphs to which Theorem 12 may apply but Theorem 13 may not. Let $G=\left(K_{r} \cup K_{r}\right) \vee K_{1}$ of order $n:=2 r+1$, where $r \geq 4$. Surely, the graph $G$ is traceable. By a little computation, $\mu(G)$ is the largest root of the polynomial $f(x)=x[x-(r-1)]-2 r$ and $q(G)$ is the largest root of the polynomial $g(x)=[x-(2 r-1)](x-2 r)-2 r$. Hence,

$$
\begin{aligned}
& \mu(G)=\frac{r+1+\sqrt{r^{2}+6 r+1}}{2}<\sqrt{4 r^{2}-8 r+6} \\
& =\sqrt{(n-3)^{2}+2} \text {, } \\
& q(G)=4 r-\frac{1}{4}>\frac{(2 r-1)^{2}+2}{r}=\frac{2(n-2)^{2}+4}{n-1} .
\end{aligned}
$$

So, we can apply Theorem 12 but not Theorem 13 for $G$ to be traceable.

\section{Signless Laplacian Spectral Radius in Hamilton-Connected Graphs}

For a graph $G$ of order $n$, Erdös and Gallai [14] prove that if

$$
d_{G}(u)+d_{G}(v) \geq n+1,
$$

for any pair of nonadjacent vertices $u$ and $v$, then $G$ is Hamilton-connected.

The idea for the closure of a graph can be found in [7]. For a positive integer $k$, the $k$-closure of a graph $G=(V, E)$, denoted by $\mathscr{C}_{k}(G)$, is a graph obtained from $G$ by successively joining pairs of nonadjacent vertices $u \in V$ and $v \in V$, whose degree sum is at least $k$ until no such pairs remain. By the definition of the $k$-closure of $G$, we have that $d_{\mathscr{C}_{k}(G)}(u)+$ $d_{\mathscr{C}_{k}(G)}(v) \leq k-1$ for any pair of nonadjacent vertices $u \in V$ and $v \in V$ of $\mathscr{C}_{k}(G)$.

Lemma 15 (see [7]). Let $G$ be a graph of order $n$. Then, $G$ is Hamilton-connected if and only if $\mathscr{C}_{n+1}(G)$ is Hamiltonconnected.
Lemma 16. Let $G$ be a simple graph with degree sequence $\left(d_{G}\left(v_{1}\right), d_{G}\left(v_{2}\right), \ldots, d_{G}\left(v_{n}\right)\right)$, where $d_{G}\left(v_{1}\right) \leq d_{G}\left(v_{2}\right) \leq \cdots \leq$ $d_{G}\left(v_{n}\right)$ and $n \geq 3$. Suppose that there is no integer $k \leq n / 2$ such that $d_{G}\left(v_{k-1}\right) \leq k$ and $d_{G}\left(v_{n-k}\right) \leq n-k$. Then, $G$ is Hamiltonconnected.

Proof. Let $\bar{H}=\mathscr{C}_{n+1}(G)$ be the $(n+1)$-closure of $G$. Next, we will prove that $\bar{H}$ is a complete graph; then the result follows according to (29). To the contrary, suppose that $\bar{H}$ is not a complete graph, and let $u$ and $v$ be two nonadjacent vertices in $\bar{H}$ with

$$
d_{\bar{H}}(u) \leq d_{\bar{H}}(v)
$$

and $d_{\bar{H}}(u)+d_{\bar{H}}(v)$ being as large as possible. By the definition of $\mathscr{C}_{n+1}(G)$, we have

$$
d_{\bar{H}}(u)+d_{\bar{H}}(v) \leq n .
$$

Denote by $S$ the set of vertices in $V \backslash\{v\}$ which are nonadjacent to $v$ in $\bar{H}$. Denote by $T$ the set of vertices in $V \backslash\{u\}$ which are nonadjacent to $u$ in $\bar{H}$. Then,

$$
|S|=n-1-d_{\bar{H}}(v), \quad|T|=n-1-d_{\bar{H}}(u) .
$$

Furthermore, by $d_{\bar{H}}(u)+d_{\bar{H}}(v)$ being as large as possible, each vertex in $S$ has degree at most $d_{\bar{H}}(u)$ and each vertex in $T \cup\{u\}$ has degree at most $d_{\bar{H}}(v)$. Let $k:=d_{\bar{H}}(u)$. According to (31) and (32), we have that $|S|=n-1-d_{\bar{H}}(v) \geq d_{\bar{H}}(u)-1=k-1$, $|T|+1=n-1-d_{\bar{H}}(u)+1=n-d_{\bar{H}}(u)=n-k$. Then $\bar{H}$ has at least $k-1$ vertices of degree not exceeding $k$ and at least $n-k$ vertices of degree not exceeding $n-k$. Because $G$ is a spanning subgraph of $\bar{H}$, the same is true for $G$; that is, $d_{G}\left(v_{k-1}\right) \leq k$ and $d_{G}\left(v_{n-k}\right) \leq n-k$. Because $k \leq n / 2$ by (30) and (31), this is contrary to the hypothesis. So we have that the $(n+1)$-closure $\bar{H}$ of $G$ is indeed complete graph and hence that $G$ is Hamilton-connected by (29).

We write $K_{n-1}+e+e^{\prime}$ for $K_{n-1}$ together with a vertex joining two vertices of $K_{n-1}$ by edges $e, e^{\prime}$, respectively.

Lemma 17. Let $G$ be a connected graph of order $n \geq 6$. If

$$
e(G) \geq \frac{(n-1)(n-2)}{2}+2,
$$

then $G$ is Hamilton-connected unless $G \cong K_{n-1}+e+e^{\prime}$ or $G \cong \mathrm{O}_{3} \vee K_{3}$.

Proof. Suppose that $G$ is not a Hamilton-connected graph with degree sequence $\left(d_{G}\left(v_{1}\right), d_{G}\left(v_{2}\right), \ldots, d_{G}\left(v_{n}\right)\right)$, where $d_{G}\left(v_{1}\right) \leq d_{G}\left(v_{2}\right) \leq \cdots \leq d_{G}\left(v_{n}\right)$ and $n \geq 6$. By Lemma 16, 
there is integer $k \leq n / 2$ such that $d_{G}\left(v_{k-1}\right) \leq k$ and $d_{G}\left(v_{n-k}\right) \leq$ $n-k$. Since $G$ is connected, $k \geq 2$. Thus,

$$
\begin{aligned}
e(G) & =\frac{1}{2} \sum_{i=1}^{n} d_{G}\left(v_{i}\right) \\
& \leq \frac{1}{2}[(k-1) k+(n-2 k+1)(n-k)+k(n-1)] \\
& =\frac{1}{2}\left(n^{2}-2 n k+3 k^{2}-3 k+n\right) \\
& =\frac{8 n^{2}-9}{24}+\frac{3}{2}\left(k-\frac{2 n+3}{6}\right)^{2} .
\end{aligned}
$$

Because $2 \leq k \leq n / 2,(9-2 n) / 6 \leq k-(2 n+3) / 6 \leq(n-3) / 6$. Thus, if $n \geq 6$,

$$
e(G) \leq \frac{8 n^{2}-9}{24}+\frac{3}{2}\left(k-\frac{2 n+3}{6}\right)^{2} \leq \frac{(n-1)(n-2)}{2}+2 .
$$

Since $e(G) \geq(n-1)(n-2) / 2+2$, then all inequalities in the above argument should be equalities. From the last equality in (35), we have $k=2$ or $k=3$ and $n=6$. If $k=2$, by the equality in (34), $G$ is a graph with $d_{G}\left(v_{1}\right)=2, d_{G}\left(v_{2}\right)=d_{G}\left(v_{3}\right)=\cdots=$ $d_{G}\left(v_{n-2}\right)=n-2, d_{G}\left(v_{n-1}\right)=d_{G}\left(v_{n}\right)=n-1$, which implies $G \cong K_{n-1}+e+e^{\prime}$. If $k=3$ and $n=6$, by the equality in (34), $G$ is a graph with $d_{G}\left(v_{1}\right)=d_{G}\left(v_{2}\right)=d_{G}\left(v_{3}\right)=3, d_{G}\left(v_{4}\right)=$ $d_{G}\left(v_{5}\right)=d_{G}\left(v_{6}\right)=5$, which implies $G \cong O_{3} \vee K_{3}$.

Theorem 18. Let $G$ be a connected graph of order $n \geq 6$. If

$$
q(G) \geq 2(n-2)+\frac{4}{n-1}
$$

then $G$ is Hamilton-connected.

Proof. By Corollary 11 and (36), we have

$$
e(G) \geq \frac{q(G)(n-1)-(n-1)(n-2)}{2} \geq \frac{(n-1)(n-2)}{2}+2 \text {. }
$$

Suppose that $G$ is not Hamilton-connected. Then, by Lemma 17 and (37), $G \cong K_{n-1}+e+e^{\prime}$ or $G \cong O_{3} \vee K_{3}$.

If $G \cong K_{n-1}+e+e^{\prime}$. Let $X=\left(X_{1}, X_{2}, \ldots, X_{n}\right)^{T}$ be the eigenvector of $Q(G)$ corresponding to the eigenvalue $q(G)$. By (36), we know that $q(G) \neq n-3$ and $q(G) \neq n-2$. Thus, by (17), all vertices of degree $n-2$ have the same values given by $X$, say $X_{1}$, and all vertices of degree $n-1$ have the same values, say $X_{2}$. Denote by $X_{3}$ the value of the vertex of degree 2 given by $X$. Also, by (17), we have

$$
\begin{gathered}
(q(G)-(n-2)) X_{1}=(n-4) X_{1}+2 X_{2}, \\
(q(G)-(n-1)) X_{2}=(n-3) X_{1}+X_{2}+X_{3} \\
(q(G)-2) X_{3}=2 X_{2} .
\end{gathered}
$$

Transform (38) into a matrix equation $(B-q(G) \mathbf{I}) X^{\prime}=0$, where $X^{\prime}=\left(X_{1}, X_{2}, X_{3}\right)^{T}$ and

$$
B=\left[\begin{array}{ccc}
2 n-6 & 2 & 0 \\
n-3 & n & 1 \\
0 & 2 & 2
\end{array}\right]
$$

Thus, $q(G)$ is the largest root of following equation:

$$
q^{3}+(4-3 n) q^{2}+\left(2 n^{2}-2 n-8\right) q-4 n^{2}+20 n-24=0 \text {. }
$$

Let $f(x)=x^{3}+(4-3 n) x^{2}+\left(2 n^{2}-2 n-8\right) x-4 n^{2}+20 n-24$; then $f^{\prime}(x)=3 x^{2}+2(4-3 n) x+2 n^{2}-2 n-8$. Let $f^{\prime}(x)=0$; we have two values $x_{1}$ and $x_{2}$, such that $f^{\prime}\left(x_{1}\right)=f^{\prime}\left(x_{2}\right)=0$, where

$$
\begin{aligned}
& x_{1}=\frac{3 n-4-\sqrt{3 n^{2}-18 n+40}}{3}, \\
& x_{2}=\frac{3 n-4+\sqrt{3 n^{2}-18 n+40}}{3} .
\end{aligned}
$$
$x_{2}$.

Hence, $f(x)$ is strictly increasing with respect to $x$ for $x>$

Consider $f(2(n-2)+4 /(n-1))=4(n-3)^{2}\left(n^{2}-3 n+\right.$ $6) /(n-1)^{3}>0$ and $2(n-2)+4 /(n-1)>x_{2}$, which implies that $q(G)<2(n-2)+4 /(n-1)$.

If $G \cong O_{3} \vee K_{3}$. We can calculate that $q(G)=5+\sqrt{13}<$ $8.8=2(6-2)+4 /(6-1)$. Thus, in either case, we have a contradiction.

\section{Conflict of Interests}

The authors declare that there is no conflict of interests regarding the publication of this paper.

\section{Acknowledgments}

This work is jointly supported by the National Natural Science Foundation of China under Grant nos. 11071001 and 11071002, the Natural Science Foundation of Anhui Province of China under Grant no. 11040606M14, the Natural Science Foundation of Department of Education of Anhui Province of China under Grant nos. KJ2011A195 and KJ2013A196, and the Young Scientific Research Foundation of Anqing Normal College, no. KJ201307.

\section{References}

[1] M. Fiedler and V. Nikiforov, "Spectral radius and Hamiltonicity of graphs," Linear Algebra and Its Applications, vol. 432, no. 9, pp. 2170-2173, 2010.

[2] B. Zhou, "Signless Laplacian spectral radius and Hamiltonicity," Linear Algebra and Its Applications, vol. 432, no. 2-3, pp. 566$570,2010$.

[3] M. Lu, H. Q. Liu, and F. Tian, "Spectral radius and Hamiltonian graphs," Linear Algebra and Its Applications, vol. 437, no. 7, pp. 1670-1674, 2012.

[4] R. Li, "Egienvalues, Laplacian Eigenvalues, and some Hamiltonian properties of graphs," Utilitas Mathematica, vol. 88, pp. 247-257, 2012.

[5] S. Butler and F. Chung, "Small spectral gap in the combinatorial Laplacian implies Hamiltonian," Annals of Combinatorics, vol. 13, no. 4, pp. 403-412, 2010. 
[6] Y. Z. Fan and G. D. Yu, "Spectral condition for a graph to be Hamiltonian with respect to normalized Laplacian," http:// arxiv.org/abs/1207.6824.

[7] J. A. Bondy and V. Chvatal, "A method in graph theory," Discrete Mathematics, vol. 15, no. 2, pp. 111-135, 1976.

[8] G. Hendry, "Extending cycles in bipartite graphs," Journal of Combinatorial Theory B, vol. 51, no. 2, pp. 292-313, 1991.

[9] V. Chvátal, “On the Hamilton's ideal's," Journal of Combinatorial Theory B, vol. 12, pp. 163-168, 1972.

[10] W. So, "Commutativity and spectra of Hermitian matrices," Linear Algebra and Its Applications, vol. 212-213, no. 15, pp. 121129, 1994.

[11] K. Ch. Das, "The Laplacian spectrum of a graph," Computers \& Mathematics with Applications, vol. 48, no. 5-6, pp. 715-724, 2004.

[12] K. Ch. Das, "Maximizing the sum of the squares of the degrees of a graph," Discrete Mathematics, vol. 285, no. 1-3, pp. 57-66, 2004.

[13] L. H. Feng and G. H. Yu, "On three conjectures involving the signless Laplacian spectral radius of graphs," Publications de l'Institut Mathematique, vol. 85, no. 99, pp. 35-38, 2009.

[14] P. Erdös and T. Gallai, "On maximal paths and circuits of graphs," Acta Mathematica Hungarica, vol. 10, no. 3, pp. 337356, 1959 . 


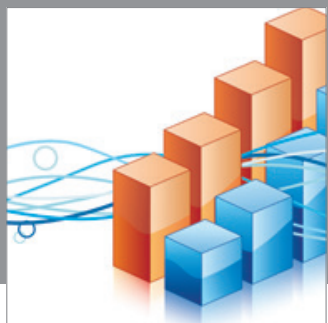

Advances in

Operations Research

mansans

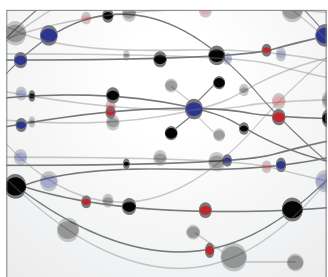

The Scientific World Journal
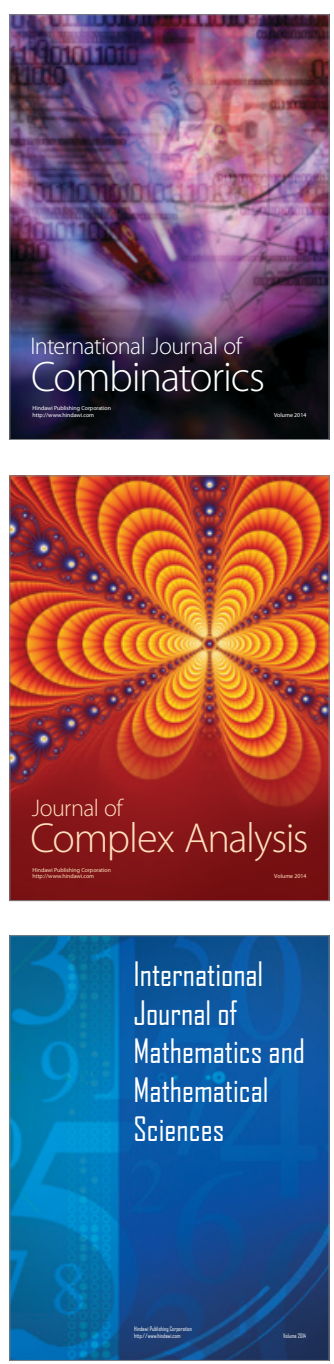
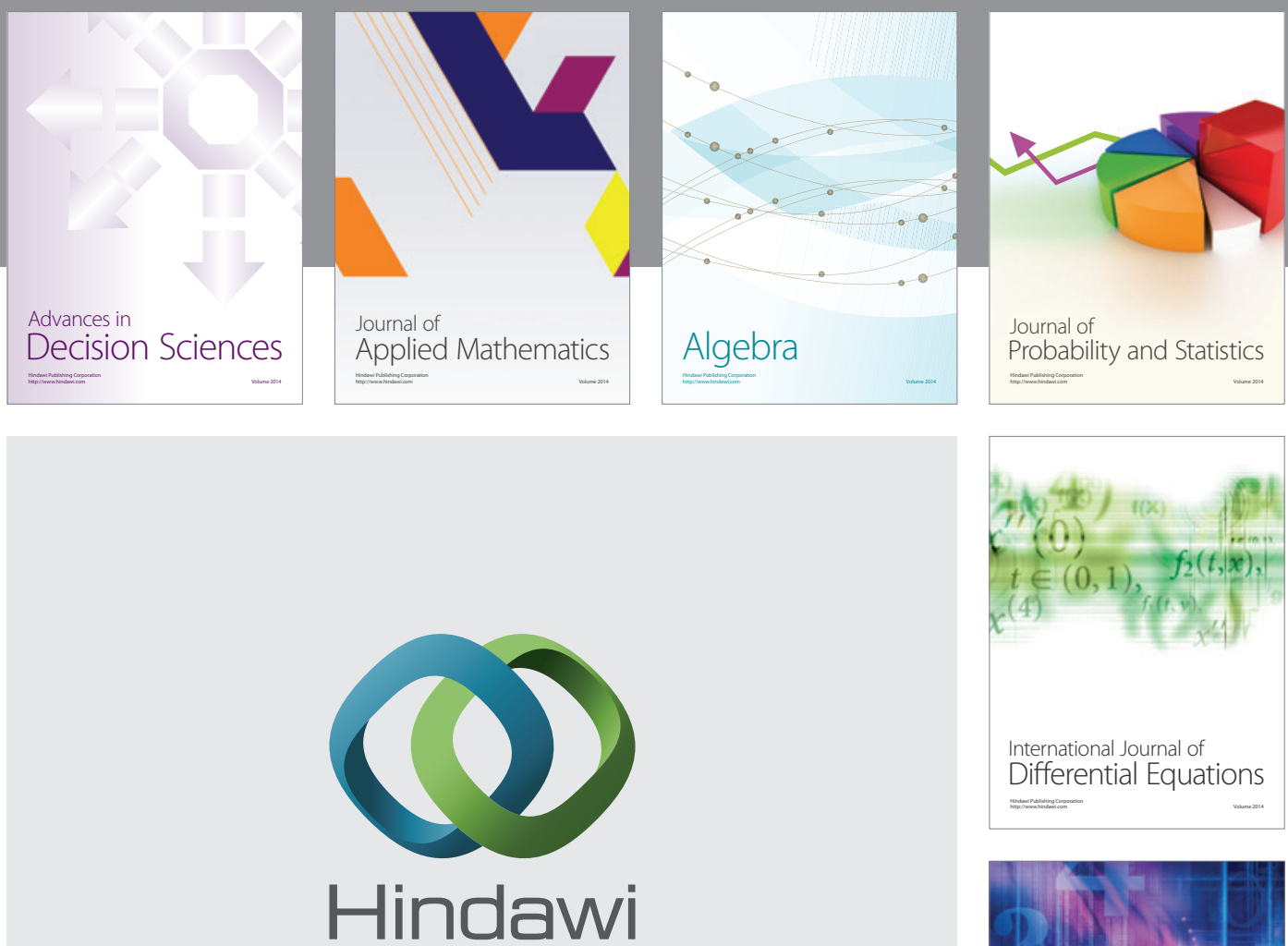

Submit your manuscripts at http://www.hindawi.com
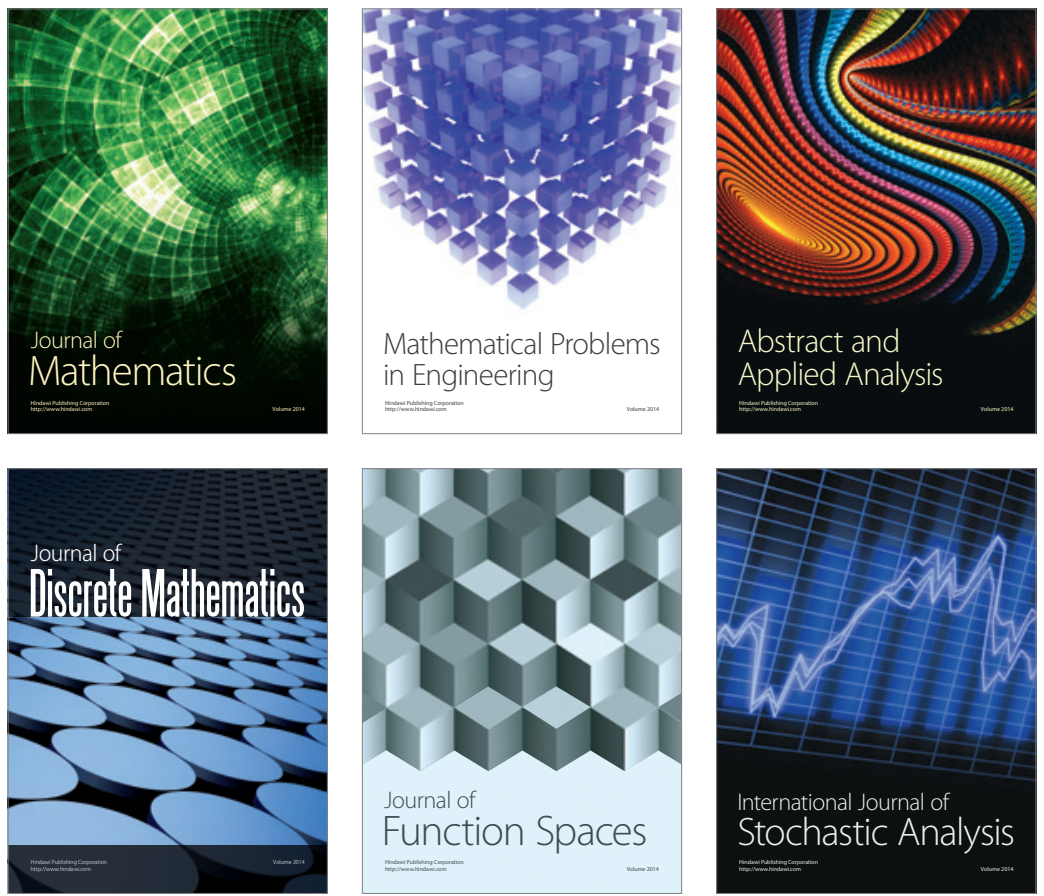

Journal of

Function Spaces

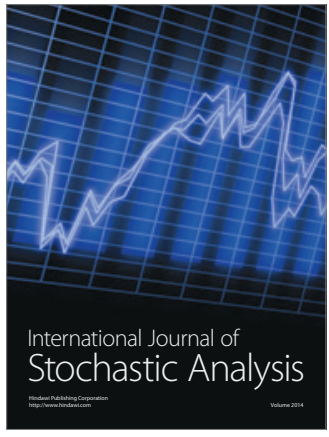

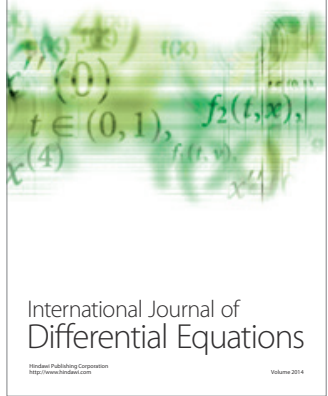
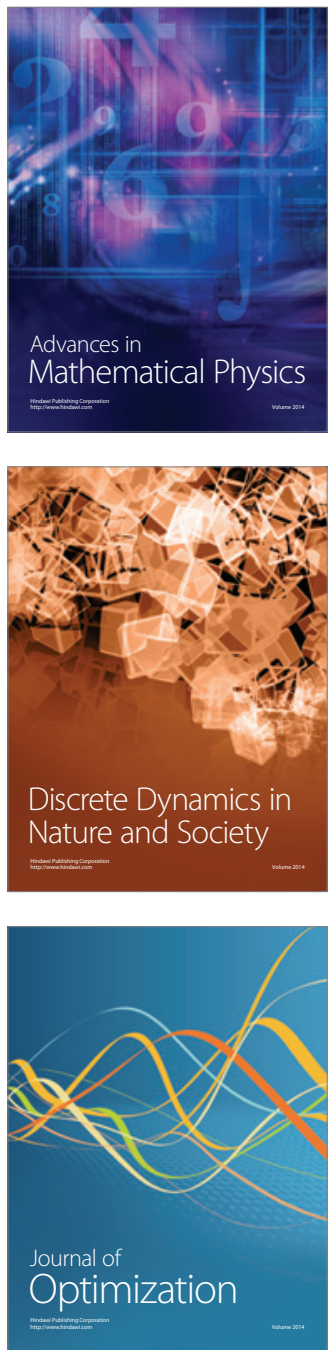